\title{
Prólogo \\ La ciberconexión como cultura mediática. Redes sociales, competencia mediática, ciudadanía
}

\author{
Ignacio Aguaded ${ }^{1}$, Águeda Delgado-Ponce ${ }^{2}$ \\ ${ }^{1}$ Programa Interuniversitario de Doctorado en Comunicación (UHU, US, UMA, UCA) \\ ${ }^{2}$ Universidad de Huelva y Universidad Internacional de Andalucía (UNIA)
}

Los entornos comunicativos mediados por las tecnologías se han convertido en el principal espacio para la interacción, la información o la difusión de contenidos. Estos entornos no solo conforman los actuales ámbitos sociales donde se relacionan las personas, sino que a su alrededor se desarrolla toda una cultura mediática caracterizada por la ciberconexión. A esto, se une la confluencia que han tenido estas redes sociales en el último año, caracterizado por la pandemia por Covid-19, donde se han dado toda una serie de propuestas, acciones y llamamientos a la participación ciudadana y la colaboración que han ampliado las fronteras de estos medios sociales. Los confinamientos nacionales, locales o individuales a los que han estado abocadas muchas personas, la teleformación y la educación a distancia han supuesto un mayor flujo de datos e informaciones a través de Internet, aunque también han mostrado las debilidades en competencia mediática de los ciudadanos.

En este contexto, es una acuciante necesidad la reflexión, el análisis, la investigación de estas transformaciones cibercomunicativas para entender mejor el mundo en el que vivimos, ofreciendo propuestas prácticas y reales que favorezcan el empoderamiento de la ciudadanía. En este sentido, esta edición especial de la Revista Media Education. Study, ricerca, buone pratiche se centra en los cambios que se están llevando a cabo a raíz de la ciberconexión, tanto en las formas comunicativas como desde la participación y la educación; así como las implicaciones de estos escenarios y la ineludible formación en competencia mediática.

Los artículos recogidos en este monográfico tienen en común la atención a estos aspectos desde diversos puntos de vista ofreciendo un amplio panorama de la situación actual desde distintos contextos.
El trabajo que abre este número especial, Tiktokers y objetivación sexual de género en retos musicales, de las autoras Arantxa Vizcaíno y Simona Tirocchi, nos acerca los nuevos ciudadanos del mundo digital, los prosumers, consumidores y productores de contenidos que surgen en los actuales entornos comunicativos. Concretamente, se centran en la red social TikTok, que cada vez está cobrando más importancia en el panorama mediático, y analizan la objetivación sexual en los vídeos musicales que generan estos usuarios. Siguiendo con las redes sociales, pero desde un punto de vista educativo, el artículo de Livia Figalli, Educare gli adolescenti ai social network. Una ricerca esplorativa su un campione di progetti nelle scuole italiane. Tendenze e modelli di analisi, aborda las intervenciones formativas llevadas a cabo con adolescentes en la última década para establecer unas pautas en el desarrollo de proyectos encaminados a la atención de los problemas que pueden causar el abuso de las redes sociales en los jóvenes y dotar a estos de las herramientas necesarias.

Las relaciones entre las tecnologías, la importancia de la educación y los adolescentes, cuyo espacio vital son los entornos mediáticos, son también estudiados por Antonia Cava, Assunta Penna, Debora Pizzimenti Onlife: adolescenti tra narrazioni e identità a través de una investigación empírica que mide los usos habituales de los medios, haciendo hincapié en aspectos tan relevantes en estas edades como la autorrepresentación y la identidad.

Los siguientes dos artículos tienen en común la atención a la alfabetización mediática del profesorado. Por una parte, Giuseppa Cappuccio y Giuseppa Compagno, en Developing support teachers' digital competencies for an inclusive citizenship, atienden al análisis de las competencias digitales de los profesores de apo- 
yo como factor crucial para el fomento de una sociedad más inclusiva, para lo que hacen uso del Marco Europeo de Competencias Digitales DIGCOMP. Esta investigación aborda en un contexto dominado por las tecnologías, a raíz de la Covid-19, la importancia de estas competencias en los docentes que tratan con alumnado con necesidades educativas especiales y que pueden quedar más apartados en este tipo de situaciones. Por otra parte, el estudio de Beatriz R. Sarmiento, María Ángeles Hernández-Prados, Noelia Carbonell-Bernal y María Cinta Aguaded-Gómez, Alfabetización del profesorado en gamificación mediada por las TIC. Estado del arte, se centra en desarrollar la alfabetización en gamificación desde una perspectiva teórica insistiendo en los beneficios de este tipo de prácticas como parte de la capacitación docente, teniendo en cuenta la profusión de experiencias gamificadas en el contexto educativo en los últimos años y las respuestas que estas han tenido en el alumnado.

Dentro de este establecimiento o análisis de un marco competencial se sitúa el artículo de Irene Melgarejo Moreno que determina las dimensiones e indicadores de la competencia mediática para la evaluación de esta en el alumnado de Educación Infantil. Este trabajo, atendiendo a las dificultades que ofrece el desarrollo de las destrezas en medios en un alumnado de esa edad, realiza una adaptación de estas dimensiones e indicadores apropiados al contexto de uso para optimizar la adquisición de dicha competencia. Siguiendo en el entorno educativo, los autores Nelly Chévez-Reynosa y María Mar Rodríguez-Rosell, en Doctorados virtuales centroamericanos: educomunicación, formación de tutores y calidad en la producción de conocimiento, sitúan el foco de su investigación en los doctorados virtuales centroamericanos y la importancia de garantizar una amplia oferta de calidad que revierta en el progreso de la ciencia. Para ello establecieron los criterios de calidad de este tipo de doctorados a partir de las entrevistas a docentes de posgrados virtuales determinando, de este modo, cómo conseguir doctorados de alto nivel.

Los últimos cuatro artículos del monográfico tienen en común la atención a los medios tradicionales, bien sobre la interrelación con la educación, la adaptación a lo digital o el estudio de problemáticas concretas. José María Merchán Bermejo, M. Cruz Alvarado, Susana de Andrés y Agustín García-Matilla con su trabajo Cine y educación: Aprender de seis décadas de buenas experiencias educomunicativas, hacen una interesante revisión de las prácticas llevadas a cabo en educación con la utilización del cine en los últimos sesenta años que pueden servir como modelo o marcar el camino a seguir para proyectos más ambiciosos o institucionales. El artículo de Diego Montalván y Jorge Galán, Supervivencia de la prensa y las radios AM en la era digital: Análisis del estado de cuatro medios de comunicación cuencanos: diarios El Mercurio y El Tiempo, y las radios La Voz del Tomebamba y Ondas Azuayas, en el período 2009 - 2020, se centra en el impacto que los avances tecnológicos y la digitalización han tenido sobre los medios de comunicación tradicionales en Ecuador, tanto desde la perspectiva de medios concretos como teniendo en cuenta la preferencia de los receptores y cómo abordar estos aspectos también desde los modelos de negocio comunicacionales. Por su parte, en el artículo Representación de mujeres en noticias sobre violencia de género

difundidas por televisión en Bolivia frente a las competencias mediáticas, Rigliana Portugal y Ignacio Aguaded, en el medio televisivo, plantean el estudio de la representación de la mujer en las noticias sobre violencia de género en distintos medios de Bolivia, privados y públicos; al mismo tiempo que analizan la competencia mediática de mujeres estudiantes de Comunicación Social, centrándose en las noticias sobre violencia de género, determinando así la necesidad de formación en este sentido. Por último, cerrando este número especial encontramos el artículo de Andrea Villarrubia-Martínez y Águeda Delgado-Ponce, Contenidos infantiles convergentes, identidad latinoamericana y los desafíos de las televisiones públicas, que realiza un análisis de la diversidad en los programas infantiles que podemos encontrar en plataformas digitales públicas de Chile y aborda las nuevas formas de consumo audiovisual generadas con la digitalización y amplificadas por la pandemia.

En definitiva, este monográfico hace un repaso por las distintas perspectivas que presenta el panorama mediático generado por la proliferación de las redes sociales, por la ciberconexión, planteando, asimismo, la importancia que tiene en este contexto y el momento actual en el que estamos la atención a una educación en medios que dote a los ciudadanos (niños, adolescentes, profesores...) de las competencias necesarias para desenvolverse eficazmente en este mundo digital en continuo cambio. 


\title{
Editoriale \\ La cyberconnessione come cultura dei media. Social network, competenza mediale, cittadinanza
}

\author{
Ignacio Aguaded ${ }^{1}$, Águeda Delgado-Ponce ${ }^{2}$ \\ ${ }^{1}$ Programa Interuniversitario de Doctorado en Comunicación (UHU, US, UMA, UCA) \\ ${ }^{2}$ Universidad de Huelva y Universidad Internacional de Andalucía (UNIA)
}

Gli ambienti di comunicazione mediati dalle tecnologie sono diventati lo spazio principale per l'interazione, l'informazione o la diffusione dei contenuti. Questi ambienti non solo costituiscono gli attuali ambienti sociali in cui le persone interagiscono, ma intorno a loro si è sviluppato un'intera cultura dei media basata sulle connessioni online. A ciò si aggiunge il ruolo esercitato dai social network nell'ultimo anno, quello della pandemia Covid-19, nel corso del quale si sono susseguite tutta una serie di proposte, iniziative e bandi di partecipazione e collaborazione cittadina che ne hanno allargato i confini dei social network. I confini nazionali, locali o individuali a cui molte persone sono state costrette, la tele-formazione e l'istruzione a distanza hanno portato a un maggiore flusso di dati e informazioni attraverso Internet, sebbene abbiano anche mostrato evidente lacune nella competenza mediale dei cittadini.

In questo contesto, la riflessione, l'analisi e l'indagine di queste trasformazioni cibercomunicative sono urgentemente necessarie per comprendere meglio il mondo in cui viviamo, offrendo proposte pratiche e reali che favoriscano l'empowerment dei cittadini. Questo numero speciale della rivista si concentra per l'appunto su questi cambiamenti sia nelle forme comunicative che nella partecipazione dei cittadini; così come sulle implicazioni che questi scenari producono sulla formazione alla competenza mediale.

Gli articoli raccolti in questo numero monográfico hanno in comune l'attenzione a questi aspetti da diversi punti di vista, offrendo un ampio panorama della situazione attuale da diversi contesti geografici e disciplinari.

Il lavoro che apre questo numero speciale, Tiktokers y objetivación sexual de género en retos musicales, di Arantxa Vizcaíno-Verdù e Simona Tirocchi, ci avvicina ai nuovi cittadini del mondo digitale, i prosumer, consumatori e produttori di contenuti che sorgono in ambienti comunicativi attuali. Nello specifico, si concentrano sul social network TikTok, che sta acquisendo sempre più importanza nella scena dei media, e analizzano l'oggettivazione sessuale nei video musicali generati da questi utenti. Continuando con i social network, ma da un punto di vista educativo, l'articolo di Livia Figalli, Educare gli adolescenti ai social network. Una ricerca esplorativa su un campione di progetti nelle scuole italiane. Tendenze e modelli di analisi, affronta gli interventi formativi effettuati con gli adolescenti nell'ultimo decennio per stabilire delle linee guida nello sviluppo di progetti volti ad affrontare le problematiche causate dall'abuso dei social network nei giovani.

Il rapporto tra tecnologia, educazione e adolescenti, i cui spazi di vita sono sempre più online, sono studiati anche da Antonia Cava, Assunta Penna, Debora Pizzimenti in Onlife: adolescenti tra narrazioni e identità attraverso una ricerca empirica che misura gli usi abituali dei media, sottolineando aspetti rilevanti per questa fascia di età quali l'autorappresentazione e l'identità.

I due articoli seguenti hanno in comune l'attenzione alla formazione digitale degli insegnanti. Il primo, Developing support teachers' digital competencies for an inclusive citizenship, scritto da Giuseppa Cappuccio e Giuseppa Compagno, riporta uno studio sulle competenze digitali dei docenti di sostegno come fattore cruciale per la promozione di una società più inclusiva, per il quale si avvalgono del Quadro Europeo delle Competenze Digitali DIGCOMP. In particolare, in un contesto dominato dalle tecnologie a causa del Covid-19, la ricerca mette in rilievo l'importanza di queste competenze negli insegnanti che si occupano di studenti con bisogni educati- 
vi speciali che possono essere ulteriormente emarginati in questo tipo di situazioni. Il secondo articolo, Alfabetización del profesorado en gamificación mediada por las TIC. Estado del arte, scritto da Beatriz R. Sarmiento, María Ángeles Hernández-Prados, Noelia CarbonellBernal e María Cinta Aguaded-Gómez, si concentra sullo sviluppo della gamification literacy da una prospettiva teorica, insistendo sui benefici di questo tipo di pratica come parte della formazione degli insegnanti, tenendo conto dell'ampia diffusione di esperienze ludiche nel contesto educativo negli ultimi anni e delle reazioni che queste hanno avuto negli studenti.

All'interno di quadro di analisi delle competenze mediali si trova l'articolo di Irene Melgarejo Moreno, Dimensiones e indicadores de la competencia mediática en Educación Infantil, nel quale si presentano le dimensioni e gli indicatori per valutare la competenza mediale nelle classi della prima infanzia. Questo lavoro, tenendo conto delle difficoltà nello sviluppo della competenza mediale in bambini così piccoli, adegua le dimensioni e gli indicatori a questo particolare contesto di uso per ottimizzare l'acquisizione di tale competenza. Continuando con il contesto educativo educativo, le autrici Nelly Chévez-Reynos e María Mar Rodríguez-Rosell nell'articolo Doctorados virtuales centroamericanos: educomunicación, formación de tutores y calidad en la producción de conocimiento, mettono al centro della loro ricerca i corsi di dottorato online del centro-America sottolineandone l'importanza al fine di garantire un miglioramento della qualità della ricerca. Per fare ciò, le autrici hanno stabilito dei criteri di qualità per questo tipo di corsi di dottorato online a partire da una serie di interviste con docenti di questi corsi, determinando così come ottenere dottorati di alto livello.

Gli ultimi quattro articoli della monografia hanno in comune l'attenzione ai media tradizionali rispetto al rapporto con l'educazione, alle forme di adattamento al mondo digitale e allo studio di problemi specifici. José María Merchán Bermejo, M. Cruz Alvarado, Susana de Andrés e Agustín García-Matilla con il loro articolo Cine y educación: Aprender de seis décadas de buenas experiencias educomunicativas, fanno un'interessante rassegna delle iniziative di educazione al cinema negli ultimi sessant'anni che possono fare da modello o segnare la via per progetti più ambiziosi o istituzionali. L'articolo di Diego Montalván e Jorge Galán, Supervivencia de la prensa y las radios $A M$ en la era digital: Análisis del estado de cuatro medios de comunicación cuencanos: diarios El Mercurio y El Tiempo, y las radios La Voz del Tomebamba y Ondas Azuayas, en el período 2009 - 2020, si concentra sull'impatto che il progresso tecnologico e la digitalizzazione hanno avuto sui media tradizionali in Ecuador, sia dal punto di vista dei singoli media che delle preferenze dei destinatari e su come affrontare questi cambiamenti anche dal punto di vista dei modelli di business. Da parte loro, Rigliana Portugal e Ignacio Aguaded, concentrandosi sul mezzo televisivo, propongono nel loro articolo Representación de mujeres en noticias sobre violencia de género difundidas por televisión en Bolivia frente a las competencias mediáticas uno studio sulla rappresentazione delle donne nelle notizie sulla violenza di genere nei diversi media boliviani, sia privati che pubblici. Inoltre, analizzano la competenza mediatica delle studentesse di Comunicazione Sociale, concentrandosi sulle notizie sulla violenza di genere, verificando così la necessità di una formazione in tal senso. Infine, chiude questo numero monografico l'articolo di Andrea Villarrubia-Martínez e Águeda DelgadoPonce, Contenidos infantiles convergentes, identidad latinoamericana y los desafíos de las televisiones públicas, in cui viene condotta un'analisi della varietà di programmi per bambini che possiamo trovare nelle piattaforme digitali pubbliche in Cile. Viene inoltre condotta un'analisi delle nuove forme di consumo audiovisivo nate con la digitalizzazione e amplificate dalla pandemia.

In sintesi, in questo numero monografico vengono passate in rassegna diverse prospettive circa il nuovo panorama mediatico generato dalla proliferazione dei social network, dalla cyberconnessime, sottolineando anche l'importanza della media education per fornire ai cittadini (bambini, adolescenti, insegnanti...) le competenze necessarie per agire efficacemente in questo mondo digitale in continua evoluzione. 